\section{Phase-only filters codified with Burckhardt's method}

Juan Campos, Francina Turon, Maria J. Yzuel, and

\section{Santiago Vallmitjana}

Juan Campos and S. Vallmitjana are with University of Barcelona, Physics Faculty, E-08028 Barcelona, Spain; the other authors are with Autonomous University of Barcelona, Physics Department, E-08193 Bellaterra, Spain.

Received 22 January 1990.

0003-6935/90/355232-03\$02.00/0.

(C) 1990 Optical Society of America.

We show that Burckhardt's method is available to codify phase-only filters with amplitude-only variations. Correlation experimental results are given.

The main techniques for optical pattern recognition are those based on the correlation between a test and an image. Phase-only filters (POFs) have good features for the recognition process': high discrimination capability between similar objects and high sharp correlation peaks. Nevertheless, most of the graphic devices and spatial light modulators, such as CRTs, liquid crystal TVs (LCTVs), plotters, etc., can plot exclusively intensity variations, and therefore, they can only modulate the amplitude. With those kinds of devices it is not possible to obtain directly a POF. Flavin and Horner ${ }^{2}$ proposed a method to codify POFs which presents amplitude-only variations. In this work we propose another method for the amplitude encoded phase-only filters based on Burckhardt's method. ${ }^{3}$ This codification method allows phase-only filters to be written on an amplitude modulating device.

The graphic device to be used is the laser printer which presents good features for the generation of holograms by computer. ${ }^{4}$ We present experimental results of the impulse response and of the correlation obtained by using a CGH made with the proposed technique.

Burckhardt's method is based on the possibility of the decomposition of a complex value into three complex numbers with positive amplitudes and phases equal to $0,2 \pi / 3$, and $4 \pi / 3$. This is a simplification of Lee's method ${ }^{5}$ which has four components with phases equal to $0, \pi / 2, \pi$, and $3 \pi / 2$. Then, the transmission of each pixel on the filter, $A \exp (j \Phi)$, is replaced by the following equation:

$$
A \exp (j \Phi)=A_{0}+A_{1} \exp (j 2 \pi / 3)+A_{2} \exp (j 4 \pi / 3),
$$

where $A_{0}, A_{1}$, and $A_{2}$ are real and positive numbers. The decomposition of Eq. (1) is not the only one. To obtain the experimental results we used the decomposition suggested by Burckhardt: the minor component $A_{0}, A_{1}$, or $A_{2}$ becomes equal to zero in each pixel. ${ }^{6}$

Each pixel of the filter is represented in the hologram by a cell with three apertures with transmissions $A_{0}, A_{1}$, and $A_{2}$. By illuminating the hologram with an inclined beam in such a way that the phase variation between the two sides of a cell is equal to $2 \pi$, the complex amplitude $A \exp (j \Phi)$ is obtained. The transmission of a hologram used as a matched filter is the FT of the object to be detected:

$$
H(\mu, \gamma)=A(\mu, \gamma) \exp [j \Phi(\mu, \gamma)] \text {. }
$$

The POF is generated by setting the amplitude $A(\mu, \gamma)$ equal to one:

$$
H_{F}(\mu, \gamma)=\exp [j \Phi(\mu, \gamma)] \text {. }
$$
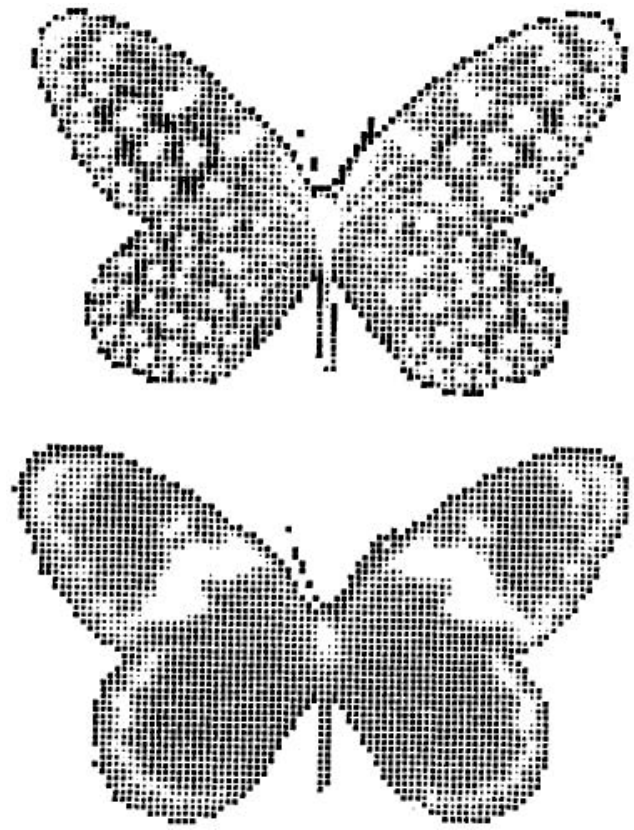

Fig. 1. Scene used in the detection process. The target to be recognized is the upper butterfly (B1).

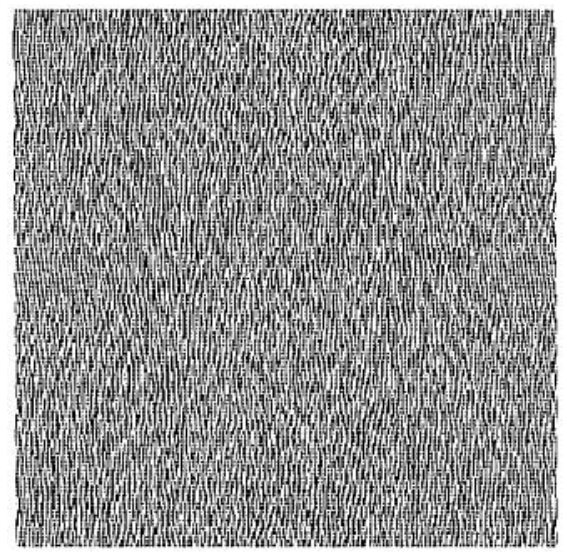

Fig. 2. Phase-only filter of $\mathrm{B} 1$ printed by a laser printer.

This filter can be coded with the same method presented above, and in this way, a POF with amplitude-only variation is obtained.

A laser printer with a resolution of $300 \mathrm{dpi}$ is used to plot the hologram. If the object has $128 \times 128$ pixels, a cell of 18 $\times 18$ dots is allowed as the maximum size when the drawing of the hologram is $\sim 7.5 \times 7.5 \mathrm{in}^{2}$. Each of the cell apertures will contain $18 \times 6=108$ dots. The transmission in amplitude $A_{\mathrm{i}}$ of every aperture depends on the number of blocked out dots. Devices that can give grey levels, such as a LCTV, allow the control of the aperture transmission with the grey level.

Binary POFs can be generated with a simplification of this method. In each cell there would not be three apertures but two. Phase $\Phi=0$ is obtained by blocking out an aperture, 
and phase $\Phi=\pi$, by doing so with the other aperture. It is evident that less resolution is necessary.

The negative of the image to be used in the recognition experiments is shown in Fig. 1. The filter is obtained from the upper butterfly (B1). The POF of B1 is shown in Fig. 2. To make the filter, a high resolution and high contrast commercial film was used. The negative of Fig. 2 was sandwiched in a liquid gate to reduce the phase variations introduced by the film. The impulse response from the filter is shown in Fig. 3. Figure 3(a) corresponds to the simulation result and Fig. 3(b) to the experimental result. An edge enhancement has been produced because the high frequencies have been powered by making the modulus of the FT of the object constant. The experimental result [Fig. 3(b)] was obtained by illuminating the hologram (Fig. 2) perpendicularly with a parallel beam. The B1 reconstruction appears in the first diffraction order. By comparing Figs. 3(a) and (b), it is possible to observe the agreement between the theoretical prediction and the experimental result.

The correlation results of the POF with the image given in Fig. 1 are shown in Fig. 4. Figure 4(a) represents the computer simulation, and Fig. 4(b) the experimental results. To obtain the optical correlation a convergent correlator was used to match the sizes of the FT of the scene and the filter. The scene reconstruction [Fig. 4(b)] also appears on the correlation plane due to the correlation between the scene and the delta function at the origin of the impulse response [Fig. 3(b)]. Figure 4 shows the good capacity of this kind of filter for discriminating between similar objects and the detectability of the correlation peak (sharp and energetic peak) which are fundamental features of POF's. The codifying method implies that the efficiency is not equal to $100 \%$

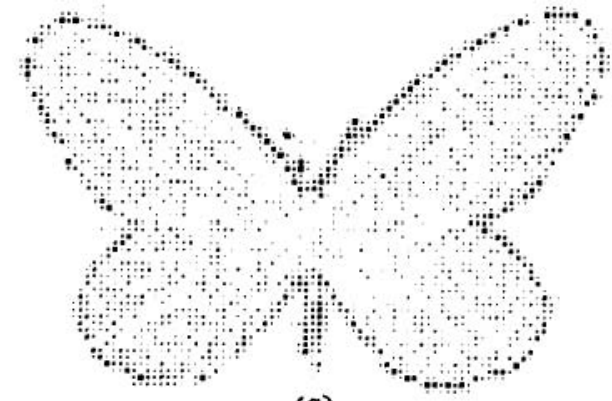

(a)

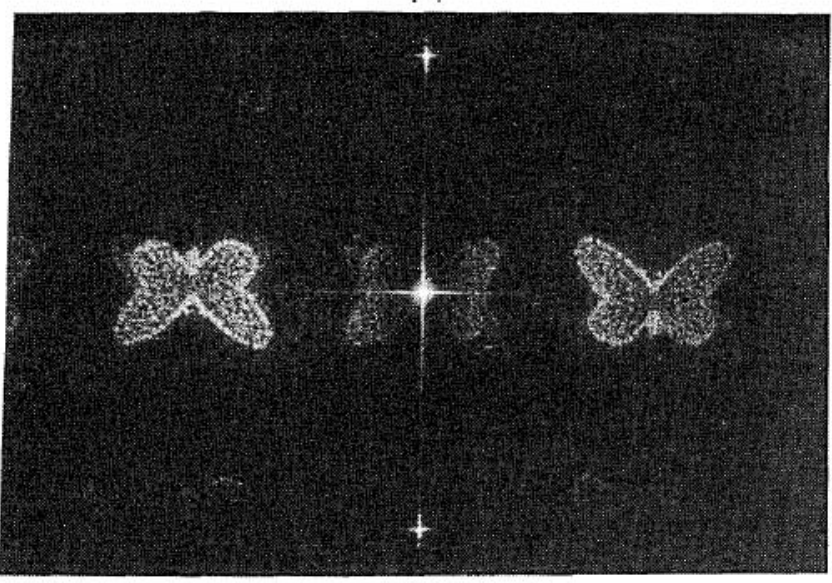

(b)

Fig. 3. (a) Result of the computer simulation of the filter impulse response and (b) optical impulse response of the filter. because the filter is not completely transparent. Nevertheless, that efficiency is greater than that of the classical matched filter codified with this method.

It has been shown that the amplitude encoded POF proposed in this paper gives good results used for pattern recognition, because very clearly detectable peaks and high discrimination ability is obtained. Moreover, the laser printer has sufficient resolution to plot the filters. These results show that the quantization does not make appearance degradations in the correlation peak.
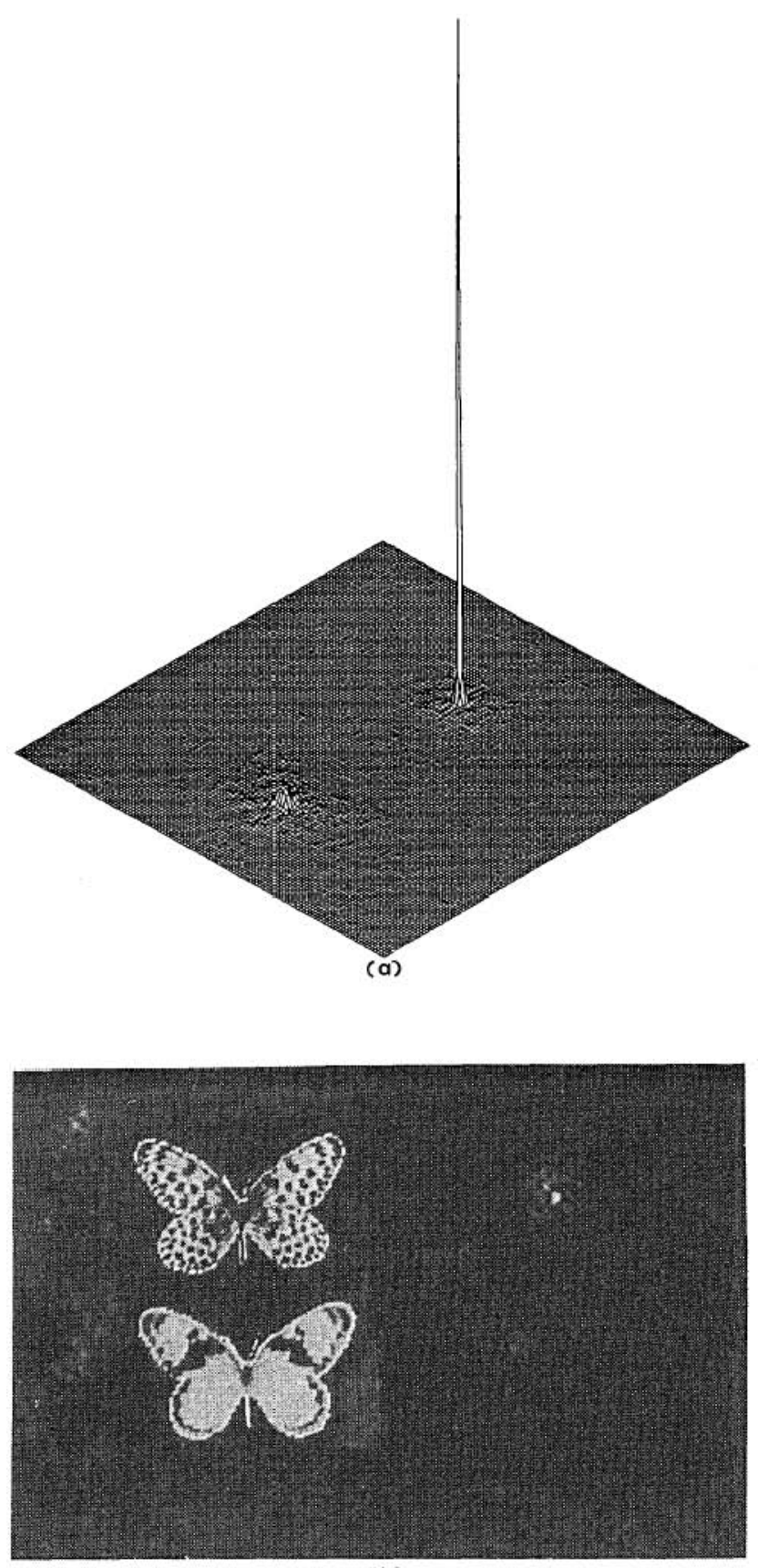

(b)

Fig. 4. (a) Computer simulation of the cross correlation and (b) optical correlation obtained with the POF codified with the proposed method. 
This work was carried out with the financial support of the DGICYT (Direccion General de Investigacion Cientifica y Tecnica), project PB87-0779.

\section{References}

1. J. L. Horner and P.D. Gianino, "Phase-Only Matched Filtering," Appl. Opt. 23, 812-816 (1984).

2. M. A. Flavin and J. L. Horner, "Amplitude Encoded Phase-Only Filters," Appl. Opt. 28, 1692-1696 (1989).
3. C. B. Burckhardt, "A simplification of Lee's Method of Generating Holograms by Computer," Appl. Opt. 9, 1949 (1970).

4. A. J. Lee and D. P. Casasent, "Computer Generated Hologram Recording Using a Laser Printer," Appl. Opt. 26, 136-138 (1987).

5. W. H. Lee, "Sampled Fourier Transform Hologram Generated by Computer," Appl. Opt. 9, 639-643 (1970).

6. H. O. Bartelt and K. D. Forster, "Computer Generated Holograms with Reduced Phase Errors," Opt. Commun. 26, 12-16 (1978). 